\title{
Optic disc measurements using the Heidelberg Retina Tomograph in amblyopia
}

This article was published in the following Dove Press journal:

Clinical Ophthalmology

13 September 2010

Number of times this article has been viewed

\author{
Atsushi Miki ${ }^{1,2}$ \\ Motohiro Shirakashi' \\ Kiyoshi Yaoeda' \\ Yu Kabasawa' \\ Satoshi Ueki' \\ Mineo Takagi' \\ Haruki Abe' \\ 'Department of Ophthalmology, \\ Niigata University Graduate School \\ of Medical and Dental Sciences, \\ Niigata; ${ }^{2}$ Department of \\ Ophthalmology, Kawasaki Medical \\ School, Okayama, Japan
}

Purpose: To investigate the characteristics of optic disc parameters in amblyopic eyes in which retinal involvement is uncertain.

Methods: A total of 44 patients with a history of unilateral amblyopia (27 patients with persistent amblyopia and 17 patients with resolved amblyopia) were examined using the Heidelberg Retina Tomograph (HRT) II. Parameters examined included disc area, cup area, cup volume, rim area, rim volume, cup-to-disc area ratio, and mean retinal nerve fiber layer thickness.

Results: In patients with persistent amblyopia, the amblyopic eyes were significantly more hyperopic than the fellow eyes. In the HRT parameters, there were no significant differences between the amblyopic and fellow eyes. In addition, after adjusting for refraction, the presence of strabismus, and the disc area, there was no significant difference in any HRT parameter between the amblyopic eyes of patients with persistent amblyopia and the previously amblyopic eyes of patients with resolved amblyopia.

Conclusions: We did not find any strong evidence for the deformity of the optic disc of amblyopic eyes.

Keywords: Heidelberg Retina Tomograph, amblyopia, optic disc, retinal nerve fiber layer thickness

\section{Introduction}

Amblyopia is a visual disorder characterized by a subnormal visual acuity and contrast sensitivity in one or both eyes, caused by either visual deprivation or abnormal binocular interaction. ${ }^{1}$ Amblyopia has generally been considered to be due to the abnormal development of the visual cortex in children. ${ }^{2}$ Histologic and functional studies of the lateral geniculation nucleus (LGN) have shown that amblyopia also affects the $\mathrm{LGN}^{3-7}$ although some previous studies investigating the function of the LGN of amblyopic monkeys have indicated the contrary. ${ }^{8}$ While previous studies have shown little evidence of retinal involvement in amblyopia, ${ }^{2,9}$ recent studies of the optic discs in amblyopia have suggested that anomalous optic discs might be found. ${ }^{10-12}$ Therefore, it is still unclear whether or not the retina is entirely normal in amblyopia.

The Heidelberg Retina Tomograph (HRT), a cofocal scanning laser ophthalmoscopic device, has been used to evaluate quantitatively the three-dimensional surface topography of the optic nerve head and the surrounding nerve fiber layer. ${ }^{13-15}$ HRT can provide objective measurements of the optic nerve head, such as cup-to-disc ratio, cup volume, rim area, and thickness of the nerve fiber layer. Therefore, HRT may be an ideal device by which to analyze the optic nerve head in amblyopic eyes.
Correspondence: Atsushi Miki

Department of Ophthalmology, Kawasaki Medical School, 577, Matsushima, Kurashiki, Okayama 701-0192, Japan

Tel +8 I 86462 I I । I

Fax +8I 864630923

Email amiki@tc5.so-net.ne.jp 
In this study, we used the HRT, optic disc morphology, and retinal nerve fiber layer thickness (RNFLT) in unilateral amblyopia in order to investigate whether or not there are any differences in these measurements between amblyopic and fellow eyes. We also compared patients whose amblyopia had improved and those whose amblyopia had failed to improve.

\section{Materials and methods}

We examined 48 patients with a history of amblyopia or who were receiving amblyopia treatment. The patients enrolled in this study had amblyopia caused by strabismus, anisometropia, or both. All of the patients were treated for their amblyopia with refractive correction and patching of the sound eye or atropine instillation in the sound eye when necessary (at least once). Some of the patients were not fully compliant with the prescribed treatment or had been found too late for treatment. The data from four patients were excluded because of poor image quality. Thus, in the end, we analyzed data from 44 patients. These patients were divided into two groups, ie, 54 eyes of 27 patients with persistent amblyopia (13 male and 14 female, aged 6-30 [10.8 \pm 5.9$]$ years when the images were taken) and 34 eyes of 17 patients who had recovered from amblyopia (six male and 11 female, whose ages ranged 5 to 15 years $(8.2 \pm 2.7$ years). Resolved amblyopia was defined as improvement in visual acuity of the amblyopic eye to 20/20 or better. All of the patients underwent a complete eye examination including cycloplegic refraction. There were no statistically significant differences in age $(P=0.059$ unpaired $t$-test), gender $(P=0.335$, Fisher's Exact test $)$, or refractive error $(P=0.53)$ for amblyopic eyes; $P=0.432$ for fellow eyes (unpaired $t$-test) distributions between persistent and resolved amblyopia. The mean final best-corrected visual acuities of the amblyopic eye in persistent and resolved amblyopia were 20/50 (range 20/200-20/30) and 20/20 (range 20/20-20/16), respectively. Both of the mean final best-corrected visual acuities of the fellow eye in persistent and resolved amblyopia were 20/16 (ranged from 20/20 to 20/16). All patients were followed up for more than five years in order to ascertain if the improvement in visual acuity had reached a plateau.

In each patient, optic disc topography was examined using HRT II (version 3.0, Heidelberg Engineering, GmbH, Heidelberg, Germany). Informed consent was obtained from each patient. HRT II uses a diode laser (670 nm wavelength) to scan the retinal surface sequentially in the horizontal and vertical directions on multiple focal planes. By using confocal scanning principles, a three-dimensional topographic image is constructed from a series of optical image sections at consecutive focal planes. The topographic image determined from the acquired three-dimensional image consists of $384 \times 384(147,456)$ pixels, each of which is a measurement of the surface height of the retina and the optic nerve head at the corresponding location. For every subject in this study, images were obtained through dilated pupils, with a 15-degree field of view. Three topographic images were obtained, combined, and automatically aligned to make a single mean topographic image for analysis. A contour line of the optic disc margin was drawn around the inner margin of the peripapillary scleral ring by an experienced examiner, who had been kept uninformed of all the other clinical information. Seven HRT parameters obtained using routine analysis were analyzed in terms of disc area, cup area, cup volume, cup-to-disc area ratio, rim area, rim volume, and RNFLT. Magnification errors were corrected using the subjects' refractive status and corneal curvature measurements.

The analysis was restricted to eyes that had valid optic disc measurements with HRT II. Good image quality was defined by appropriate focus, brightness, and clarity, minimal eye movement, optic disc centered in the image, and a standard deviation of the mean topographic image $<30 \mu \mathrm{m}$. Eyes in which good quality images could not be obtained were excluded from the analysis.

SPSS software (v. 17.0 J; SPSS Japan, Inc., Tokyo, Japan) and MedCalc version 10.1 (MedCalc Software bvba, Mariakerke, Belgium) were used to perform the statistical analysis. A paired $t$-test was used to assess differences in parameters between amblyopic (previously or persistently) and fellow eyes. An unpaired $t$-test was used to assess differences in parameters between amblyopic eyes of patients with persistent amblyopia and previously amblyopic eyes of patients with resolved amblyopia. Logistic regression analysis was applied to adjust for effects of refraction, presence of strabismus, and disc area. Because of the large number of tests, the Bonferroni correction for multiple comparisons was also applied.

\section{Results}

In patients with persistent amblyopia, the spherical equivalent refractive error in the amblyopic eyes was significantly larger $(P<0.001)$ than that in the fellow eyes, ie, the amblyopic eyes were significantly more hyperopic (see Table 1). RNFLT in amblyopic eyes was slightly thinner than that in fellow eyes $(P=0.022)$, but the difference was not significant after Bonferroni correction. There were no significant differences between amblyopic and fellow eyes in other HRT parameters. In patients with resolved amblyopia, there was no significant difference in refraction between previously amblyopic and fellow eyes. Also, there was no significant difference in each HRT parameter between the two eyes.

Rim volume in amblyopic eyes of patients with persistent amblyopia was greater than that in previously amblyopic eyes of patients with resolved amblyopia, but the $P$ value 
Table Comparison of refraction and Heidelberg Retina Tomography parameters

\begin{tabular}{|c|c|c|c|c|c|c|}
\hline \multicolumn{7}{|c|}{ Comparison between amblyopic and fellow eye } \\
\hline & \multicolumn{3}{|c|}{ Resolved amblyopia (34 eyes of 17 subjects) } & \multicolumn{3}{|c|}{ Persistent amblyopia (54 eyes of 27 subjects) } \\
\hline & Fellow eye & Amblyopic eye & P* & Fellow eye & Amblyopic eye & $P *$ \\
\hline Refraction (diopters) & $1.919(1.992)$ & $2.971(3.757)$ & 0.143 & $1.387(2.272)$ & $3.742(4.074)$ & $<0.001$ \\
\hline CDAR & $0.275(0.103)$ & $0.267(0.114)$ & 0.707 & $0.24 I(0.097)$ & $0.207(0.113)$ & 0.160 \\
\hline RNFLT (mm) & $0.191(0.061)$ & $0.192(0.058)$ & 0.918 & $0.250(0.087)$ & $0.207(0.049)$ & 0.022 \\
\hline $\mathrm{DA}\left(\mathrm{mm}^{2}\right)$ & $1.967(0.386)$ & $1.915(0.383)$ & 0.567 & $2.210(0.824)$ & $2.097(0.692)$ & 0.422 \\
\hline $\mathrm{RA}\left(\mathrm{mm}^{2}\right)$ & $1.418(0.312)$ & I.39I (0.272) & 0.700 & I.652 (0.58I) & $\mathrm{I} .633(0.474)$ & 0.870 \\
\hline $\mathrm{RV}\left(\mathrm{mm}^{3}\right)$ & $0.288(0.108)$ & $0.293(0.105)$ & 0.848 & $0.426(0.180)$ & $0.389(0.162)$ & 0.386 \\
\hline $\mathrm{CA}\left(\mathrm{mm}^{2}\right)$ & $0.548(0.26 \mathrm{I})$ & $0.528(0.300)$ & 0.689 & $0.557(0.377)$ & $0.459(0.349)$ & 0.127 \\
\hline $\mathrm{CV}\left(\mathrm{mm}^{3}\right)$ & $0.107(0.076)$ & $0.117(0.078)$ & $0.5 \mathrm{II}$ & $0.105(0.102)$ & $0.096(0.104)$ & $0.58 \mathrm{I}$ \\
\hline \multicolumn{7}{|c|}{ Comparison between the amblyopic eyes of resolved and persistent amblyopia } \\
\hline Refraction & $P^{\dagger}=0.532$ & & & & & \\
\hline CDAR & $P^{\dagger}=0.095$ & & & & & \\
\hline RNFLT & $P^{\dagger}=0.374$ & & & & & \\
\hline DA & $P^{\dagger}=0.326$ & & & & & \\
\hline RA & $P^{\dagger}=0.063$ & & & & & \\
\hline RV & $P^{\dagger}=0.037$ & & & & & \\
\hline CA & $P^{\dagger}=0.509$ & & & & & \\
\hline $\mathrm{CV}$ & $P^{\dagger}=0.488$ & & & & & \\
\hline
\end{tabular}

Notes: Data are shown as mean (standard deviation). $P$ values are for comparison between amblyopic and fellow eyes* (paired $t$-test) and between the amblyopic eyes of resolved and persistent amblyopia ${ }^{\dagger}$ (unpaired $t$-test). Because of multiple comparisons among the seven Heidelberg Retina Tomograph parameters, Bonferroni correction was applied with a level of significance of 0.007 I. Refraction: spherical equivalent values.

Abbreviations: CDAR, cup-to-disc area ratio; RNFLT, retinal nerve fiber layer thickness; DA, disc area; RA, rim area; RV, rim volume; CA, cup area; CV, cup volume.

$(P=0.037)$ was not significant after correction for multiple comparisons (see Table 1). Logistic regression analysis adjusting for refraction, presence of strabismus, and disc area showed that there was no significant difference in each HRT parameter between the two eyes $(P \geq 0.056)$.

\section{Discussion}

In this study, we have shown that optic discs in amblyopic eyes are morphologically not significantly different from those of fellow eyes. RNFLT was slightly thinner in amblyopic eyes in fellow eyes in patients with persistent amblyopia. However, when compared with previously amblyopic eyes of patients with resolved amblyopia, the RNFLT of amblyopic eyes of patients with persistent amblyopia was not thinner.

The effects of such variables as disc area, axial length, or refraction on HRT parameters must also be considered. There was a statistically significant difference in refractive error between amblyopic and fellow eyes in patients with persistent amblyopia. It is possible that refractive error has influenced measurement of RNFLT because amblyopic eyes were significantly more hyperopic. Since RNFLT has been shown to correlate negatively with refractive error in a large cross-sectional study using HRT, ${ }^{15}$ the decrease in RNFLT of amblyopic eyes might be explained by greater hyperopia in these eyes. Therefore, it seems premature to conclude that RNFLT in amblyopic eyes is different from that in normal eyes, if such an influence is not taken into account. Thus, we performed a second analysis using logistic regression analysis. However, even after adjusting for refractive errors, presence of strabismus, and disc area, there was no significant difference in each HRT parameter, including RNFLT, between amblyopic eyes of patients with persistent amblyopia and previously amblyopic eyes of patients with resolved amblyopia.

Whether or not the RNFLT values in amblyopic eyes are different from those in normal subjects is very important in elucidating the site responsible for amblyopic visual deficits. Previous studies of RNFLT have shown inconsistent results. The majority of the studies have demonstrated no significant difference between amblyopic and fellow or control eyes, ${ }^{16-24}$ although some studies have shown that RNFLT may be thicker in eyes with refractive (anisometropic) amblyopia. ${ }^{25,26}$ Yen et al hypothesized that the RNFLT is thicker in amblyopic eyes because amblyopia affects the process of postnatal reduction of ganglion cells. ${ }^{25}$ On the other hand, Duranoglu found that RNFLT was thinner in amblyopic eyes. ${ }^{27}$ However, in these studies investigating RNFLT in amblyopia, the difference in RNFLT between the eyes was quite small, at most around $10 \mu \mathrm{m}$, even when there was a significant difference. Such a small difference in RNFLT may not be clinically significant.

Do optic discs in amblyopic eyes have any deformity, such as Lempert has shown? Using optic disc images and calculations for magnification, Lempert showed that the rim 
areas and disc areas of amblyopic eyes were smaller than those of fellow eyes or normal control eyes. ${ }^{10-12} \mathrm{He}$ assumed that there is a peripheral cause for visual deficits in amblyopia. Our results did not confirm his data. We did not find any significant differences in optic disc parameters, including disc area, between amblyopic and fellow eyes. On the other hand, Duranoglu found differences in some of the HRT parameters between amblyopic and fellow or control eyes. ${ }^{27}$ However, there was no clear explanation for those findings. Atilla et al have noted that both hyperopic and amblyopic subjects had a smaller disc area and cup-disc ratio in comparison with a control group, but there was no significant difference between the hyperopic and amblyopic groups. ${ }^{19}$ They also suggested the influence of refractive errors on optic disc topography.

In conclusion, we did not find any strong evidence for deformity of the optic disc in amblyopic eyes using HRT. Our finding, together with most of those of the previous studies, will not change current practice of amblyopia treatment, but it may have an implication on future treatment targeting the sites affected by amblyopia.

\section{Disclosure}

The authors report no conflict of interest in this work.

\section{References}

1. von Noorden GK. Amblyopia: A multidisciplinary approach. Proctor lecture. Invest Ophthalmol Vis Sci. 1985;26(12):1704-1716.

2. Hess RF. Amblyopia: Site unseen. Clin Exp Optom. 2001;84(6): 321-336.

3. von Noorden GK, Crawford ML, Levacy RA. The lateral geniculate nucleus in human anisometropic amblyopia. Invest Ophthalmol Vis Sci. 1983;24(6):788-790.

4. von Noorden GK, Crawford ML. The lateral geniculate nucleus in human strabismic amblyopia. Invest Ophthalmol Vis Sci. 1992;33(9): 2729-2732.

5. Miki A, Liu GT, Goldsmith ZG, Liu C-SJ, Haselgrove JC. Decreased activation of the lateral geniculate nucleus in a patient with anisometropic amblyopia demonstrated by functional magnetic resonance imaging. Ophthalmologica. 2003;217(5):365-369.

6. Hess RF, Thompson B, Gole G, Mullen KT. Deficient responses from the lateral geniculate nucleus in humans with amblyopia. Eur J Neurosci. 2009;29(5):1064-1070.

7. Barnes GR, Li X, Thompson B, Singh KD, Dumoulin SO, Hess RF. Decreased gray matter concentration in the lateral geniculate nuclei in human amblyopes. Invest Ophthalmol Vis Sci. 2010;51(3):1432-1438.

8. Kiorpes L, McKee SP. Neural mechanisms underlying amblyopia. Curr Opin Neurobiol. 1999;9(4):480-486.

Clinical Ophthalmology

\section{Publish your work in this journal}

Clinical Ophthalmology is an international, peer-reviewed journal covering all subspecialties within ophthalmology. Key topics include: Optometry; Visual science; Pharmacology and drug therapy in eye diseases; Basic Sciences; Primary and Secondary eye care; Patient Safety and Quality of Care Improvements. This journal is indexed on Submit your manuscript here: http://www.dovepress.com/clinical-ophthalmology-journal
9. Ikeda H, Tremain KE. Amblyopia occurs in retinal ganglion cells in cats reared with convergent squint without alternating fixation. Exp Brain Res. 1979;35(3):559-582.

10. Lempert P. Optic nerve hypoplasia and small eyes in presumed amblyopia. JAAPOS. 2000;4(5):258-266.

11. Lempert $P$. The axial length/disc area ratio in anisometropic hyperopic amblyopia: A hypothesis for decreased unilateral vision associated with hyperopic anisometropia. Ophthalmology. 2004;111(2):304-308.

12. Lempert $P$. Retinal area and optic disc rim area in amblyopic, fellow, and normal hyperopic eyes: A hypothesis for decreased acuity in amblyopia. Ophthalmology. 2008;115(12):2259-2261.

13. Girkin CA, McGwin G Jr, McNeal SF, DeLeon-Ortega J. Racial differences in the association between optic disc topography and early glaucoma. Invest Ophthalmol Vis Sci. 2003;44(8):3382-3387.

14. Medeiros FA, Zangwill LM, Bowd C, Weinreb RN. Comparison of the GDx VCC scanning laser polarimeter, HRT II confocal scanning laser ophthalmoscope, and stratus OCT optical coherence tomograph for the detection of glaucoma. Arch Ophthalmol. 2004;122(6):827-837.

15. Abe H, Shirakashi M, Tsutsumi T, et al; Tajimi Study Group. Laser scanning tomography of optic discs of the normal Japanese population in a population-based setting. Ophthalmology. 2009;116(2):223-230.

16. Colen TP, de Faber JT, Lemij HG. Retinal nerve fiber layer thickness in human strabismic amblyopia. Binocul Vis Strabismus Q. 2000;15(2): 141-146.

17. Baddini-Caramelli C, Hatanaka M, Polati M, Umino AT, Susanna R Jr. Thickness of the retinal nerve fiber layer in amblyopic and normal eyes: A scanning laser polarimetry study. JAAPOS. 2001;5(2):82-84.

18. Bozkurt B, Irkec M, Orhan M, Karaagaoglu E. Thickness of the retinal nerve fiber layer in patients with anisometropic and strabismic amblyopia. Strabismus. 2003;11(1):1-7.

19. Atilla H, Batioğlu F, Erkam N. Retinal nerve fiber analysis in subjects with hyperopia and anisometropic amblyopia. Binocul Vis Strabismus $Q$. 2005;20(1):33-37.

20. Altintas O, Yuksel N, Ozkan B, Caglar Y. Thickness of the retinal nerve fiber layer, macular thickness, and macular volume in patients with strabismic amblyopia. J Pediatr Ophthalmol Strabismus. 2005;42(4):216-221.

21. Kee SY, Lee SY, Lee YC. Thicknesses of the fovea and retinal nerve fiber layer in amblyopic and normal eyes in children. Korean J Ophthalmol. 2006;20(3):177-181.

22. Dickmann A, Petroni S, Salerni A, Dell'Omo R, Balestrazzi E. Unilateral amblyopia: An optical coherence tomography study. JAAPOS. 2009;13(2):148-150.

23. Repka MX, Kraker RT, Tamkins SM, Suh DW, Sala NA, Beck RW; Pediatric Eye Disease Investigator Group. Retinal nerve fiber layer thickness in amblyopic eyes. Am J Ophthalmol. 2009;148(1):143-147.

24. Huynh SC, Samarawickrama C, Wang XY, et al. Macular and nerve fiber layer thickness in amblyopia: The Sydney Childhood Eye Study. Ophthalmology. 2009;116(9):1604-1609.

25. Yen MY, Cheng CY, Wang AG. Retinal nerve fiber layer thickness in unilateral amblyopia. Invest Ophthalmol Vis Sci. 2004;45(7):2224-2230.

26. Yoon SW, Park WH, Baek SH, Kong SM. Thicknesses of macular retinal layer and peripapillary retinal nerve fiber layer in patients with hyperopic anisometropic amblyopia. Korean J Ophthalmol. 2005;19(1):62-67.

27. Duranoglu Y. Optic nerve head topographic analysis and retinal nerve fiber layer thickness in strabismic and anisometropic amblyopia. Ann Ophthalmol (Skokie). 2007;39(4):291-295.

\section{Dovepress}

PubMed Central and CAS, and is the official journal of The Society of Clinical Ophthalmology (SCO). The manuscript management system is completely online and includes a very quick and fair peer-review system, which is all easy to use. Visit http://www.dovepress.com/ testimonials.php to read real quotes from published authors. 